\title{
Long-Term Care Insurance and Intra-Family Moral Hazard: Fixed vs Proportional Insurance Benefits
}

\author{
Justina Klimaviciute ${ }^{* \dagger}$
}

This version: September, 2016

\begin{abstract}
Pauly (1990) argues that an explanation for the low long-term care (LTC) insurance demand could be intra-family moral hazard: parents might refuse to buy insurance since it reduces children's incentives to provide care. This paper raises and explores the idea that the extent of intra-family moral hazard and the non-purchase of LTC insurance might differ when insurance benefits are fixed and when they are proportional to LTC expenditure. It shows that fixed benefits limit and might even eliminate intra-family moral hazard, while the effect of proportional benefits is at best ambiguous. Consequently, the non-purchase of insurance is less likely with fixed benefits.
\end{abstract}

JEL codes: D14, D64, G22, I11, J14.

Keywords: long-term care, long-term care insurance, intra-family moral hazard, informal care.

\section{Introduction}

Long-term care (LTC) has been increasingly becoming a "hot" topic over the last years, and its importance is predicted to grow even more in the coming decades. LTC is the care for people who are dependent on

\footnotetext{
*Toulouse School of Economics, France. Present affiliation: University of Liège, Belgium. E-mail: justina.klimaviciute@ulg.ac.be.

†'I am very grateful to Helmuth Cremer, Geir Godager, Marie-Louise Leroux, Jean-Marie Lozachmeur, Volker Meier, Pierre Pestieau, Kerstin Roeder, Emmanuel Thibault and an anonymous referee for their helpful comments, suggestions and advice. I also wish to thank the participants of the European Health Economics Workshop 2013, the Public Economic Theory Conference 2013 and the Journée Maurice Marchand des Doctorants en Economie de la Santé 2013 for their useful comments and remarks.
} 
the help of others in their basic daily activities (such as dressing, bathing, eating, etc.). It can be seen as consisting of both health and social care which can be provided both formally (by paid professional caregivers) and informally (by family members or friends), at home and in special institutions. Since the need for this kind of care is highly related with age, ${ }^{1}$ more and more attention to LTC issues is required due to the apparent population ageing in most developed countries. Indeed, the European Union has estimated that from 2007 to 2060 the number of dependent old persons in the EU27 might increase by 90 or even by 115 percent, which means that the number of dependent elderly might possibly more than double (European Commission, 2009). ${ }^{2}$ In the light of these trends, it is important to make sure that our societies are ready to successfully face the challenge. For the moment, however, a number of unresolved issues exist.

One of such issues is the so-called LTC insurance puzzle. This puzzle has been created by a surprisingly low demand for private LTC insurance. In particular, even though the potential LTC costs are large ${ }^{3}$ and the probability to become dependent is high, ${ }^{4}$ only a small fraction of individuals purchase private LTC insurance. A number of different factors potentially explaining this puzzle have been proposed in the literature. Those possible explanations range from the ones that assume perfect rationality of individuals (for instance, the high price of insurance or crowding out by the State) to the ones considering the nonpurchase of insurance as irrational (such as myopia or ignorance and denial of the issue of dependence). ${ }^{5}$ Even though it seems that none of the proposed reasons alone is able to entirely explain the puzzle, each of them might be playing some role in the issue. Therefore, being able to solve or at least reduce (some of) these potential problems might help to stimulate the market for private LTC insurance.

This paper deals with one of these potential problems. In particular, it is the problem of intra-family moral hazard, which was proposed as a possible explanation for the LTC insurance puzzle by Pauly (1990). The idea of intra-family moral hazard is that insurance owned by a parent reduces his/her

\footnotetext{
${ }^{1}$ For instance, around half of all LTC users in the OECD countries are over 80 years old (Colombo et al., 2011).

${ }^{2} \mathrm{~A} 90 \%$ increase is predicted assuming that age-specific disability rates will decline in the future, while a $115 \%$ increase is expected if these rates remain constant (European Commission, 2009).

${ }^{3}$ For instance, a nursing home stay in the U.S. costs between $\$ 40000$ and $\$ 70000$ per year, while the average cost in France is around $€ 35000$ per year (Taleyson, 2003).

${ }^{4}$ According to Kemper and Murtaugh (1991), a 65-year-old person has a probability of $43 \%$ to enter a nursing home at some time before his/her death. See also Norton (2000).

${ }^{5}$ For recent surveys of potential explanations for the puzzle, see Cremer et al. (2012), Pestieau and Ponthière (2011) and Brown and Finkelstein (2011).
} 
children's incentives to provide informal care. Indeed, children might be induced to provide care to their parents by the fact that their caregiving helps to decrease their parents' expenses on formal care and thus protects the bequest that the parents will leave to them. However, if parents have LTC insurance, the cost of formal care is (at least partly) covered by the insurer. Thus, LTC insurance also protects their future bequest ${ }^{6}$ which results in their children having less incentive to provide care. Pauly (1990) then argues that, in the fear of intra-family moral hazard, parents who prefer being taken care of by their children rather than by unknown formal caregivers might be discouraged from buying LTC insurance. While Pauly's (1990) argument is developed with little formalization, Zweifel and Strüwe (1998) provide a more rigorous model for this idea and show that it is indeed reasonable to believe that the intra-family moral hazard effect might be a cause of the non-purchase of LTC insurance. ${ }^{7}$

However, the analysis of Zweifel and Strüwe (1998) only focuses on one type of insurance benefits, namely, benefits which are proportional to the amount of LTC expenditure. ${ }^{8}$ The present paper puts forward the idea that the extent of the above described problem might be different depending on the form of insurance benefits. The motivation for this idea is the following.

The proportional benefits analyzed by Zweifel and Strüwe (1998) roughly capture the idea behind LTC insurance benefits prevalent in the U.S. where these benefits take the form of a reimbursement for the LTC expenditure the insured has, in the limit of a certain reimbursement ceiling (Duran and Taleyson, 2003). However, this is not the only type of LTC insurance benefits used in practice. For instance, private insurers in France use a substantially different benefit scheme. Insurance payments in France are of the form of a fixed monthly cash benefit which starts being paid if the insured is recognized to be dependent. This benefit does not depend on the amount of LTC expenditure and can be used by the person in the way he/she decides. ${ }^{9}$ This clearly contrasts with the U.S. model where, in order

\footnotetext{
${ }^{6}$ Pauly $(1990,1996)$ names the protection of one's bequest as the main function of LTC insurance.

${ }^{7}$ Courbage and Zweifel (2011) suggest to look at intra-family moral hazard as at a two-sided phenomenon arguing that apart from the effect of insurance on incentives for children (one side of the phenomenon), the second side exists in that parents may buy less insurance if they can rely on the caregiving effort of their children. However, the caregiving effort in their paper is modeled as a preventive one that helps to keep the parent out of a nursing home (which can be seen as preventing from a need for LTC). In this paper, I consider children's caregiving as the provision of care when their parents' LTC need has already materialized and stick to defining intra-family moral hazard as a one-sided phenomenon, namely, the decrease in the children's caregiving incentives caused by the parents' insurance coverage, as suggested by Pauly (1990).

${ }^{8}$ Zweifel and Strüwe (1996) make a comparison between LTC insurance (with proportional benefits) and trust saving which is, however, a completely different financial instrument rather than another type of insurance benefits.

${ }^{9}$ LTC insurance in the U.S. and France was originally developed on the basis of different "philosophies": the U.S. model was inspired by health insurance products whereas the French one was derived from disability insurance (Kessler, 2008). It
} 
to be reimbursed, the LTC expenditure has to come from the use of services that are approved by the insurer (Duran and Taleyson, 2003). Thus, in the American scheme the insured can only benefit from the insurance if he/she uses certain services of formal care. This is not the case with fixed insurance benefits. Therefore, intuitively it seems that the use of formal care should be encouraged much less in the case of fixed benefits, which should help to limit intra-family moral hazard. Some empirical studies reviewed below seem to suggest this idea as well.

If the effect of intra-family moral hazard is strong enough, it seems likely that the fear to distort children's incentives might offset altruistic considerations (such as intentions to ensure sufficiently large bequests to their children) that parents could have when making insurance purchase. Nevertheless, interestingly enough, this does not seem to be the case in France. In particular, a study conducted by Courbage and Roudaut (2008) finds empirical evidence that in France LTC insurance purchases are driven by altruistic motives. First, they find that the demand for LTC insurance rises with the probability of leaving a bequest, which confirms Pauly's $(1990,1996)$ argument that LTC insurance is purchased to protect one's bequest. Further, they show that LTC insurance is positively associated with the number of children and the fact of living with a partner. Finally, it is found that LTC insurance is positively associated with the probability of receiving informal care in the case of such a need in the future. These findings suggest that people are not afraid that insurance coverage will distort the caregiving incentives of their relatives. In other words, the findings seem to suggest that in France the degree of intra-family moral hazard is likely to be rather low.

For comparison, one could consider the study made by Sloan and Norton (1997) who do not find evidence for altruistic bequest motive in LTC insurance decisions in the U.S. They do not detect any relationship between stated altruism (i.e. the person's wish to leave a bequest) and demand for LTC insurance. This suggests that a parent might well be willing to leave a bequest, but still decide not to purchase LTC insurance. While it is possible that some other reasons exist, one explanation for this could be the attempt to avoid intra-family moral hazard. Therefore, one could suspect that intra-family moral hazard is a more serious problem in the U.S. than in France. While it is again possible that some other factors come into play as well, a potential explanation for this difference could be the different nature of should be noted that recently American insurance companies have started making fixed benefit contracts available as well. 
insurance benefits.

The aim of this paper is to explore more formally the roles of fixed and proportional insurance benefits in the issue of intra-family moral hazard. In particular, in the context of intra-family moral hazard, the paper compares the impact of the two benefit types on the LTC related behavior of children and eventually on the insurance purchase decisions of parents.

The model considers an elderly parent and his adult child. The parent might become dependent, in which case he places a special value on the LTC received from his child. The parent also cares about the bequest that will be left to the child; thus, he might want to purchase insurance in order to protect his bequest from LTC expenses. If the parent becomes dependent, the child chooses the amount of care she wants to provide. I consider separately the cases when the child likes and dislikes providing care to the parent. In addition to the amount of caregiving, the child's utility depends on her wealth which consists of her labour market earnings and the parent's bequest. The bequest is the channel through which the child's care provision is affected by the insurance coverage the parent has. Reasoning backwards, the first part of the analysis studies the problem of the child and compares the effects that insurance with fixed and proportional benefits has on the child's choice of caregiving. The second part explores how these different effects (anticipated by the parent) influence the parent's insurance purchase decisions.

The model is generally based on Zweifel and Strüwe (1998). However, as mentioned above, Zweifel and Strüwe (1998) analyze only proportional insurance benefits. Therefore, the case of fixed benefits is introduced in this paper and comparisons between the two types of benefits are made. In addition, Zweifel and Strüwe (1998) model the parent and the child as the principal and the agent. I do not adopt this approach here but rather follow the idea of equal treatment of both parties suggested by Courbage and Zweifel (2011). ${ }^{10}$ It should be also mentioned that, differently from Zweifel and Strüwe (1998), the present paper looks at the problem of the child from the perspective of the standard consumer's theory, which allows to identify and understand the substitution and income effects that influence the child's choice.

The paper finds that the amount of caregiving of a child who dislikes providing care is decreasing in

\footnotetext{
${ }^{10}$ However, in Courbage and Zweifel (2011) the parent's choice of insurance and the child's choice of care provision are modeled as simultaneous decisions. Here the choices are sequential since the parent has to make the insurance decision first (before one of the two possible states of nature materializes), and the child chooses the amount of care only when (and if) the state of nature with the need for LTC materializes.
} 
the parent's insurance coverage with both proportional and fixed insurance benefits. Thus, intra-family moral hazard exists with both types of benefits. However, it is also shown that the same amount of LTC insurance purchased by the parent results in more care given by the child when insurance benefits are fixed than when they are proportional. This confirms that fixed benefits indeed mitigate the phenomenon of intra-family moral hazard. On the other hand, intra-family moral hazard is never a problem with fixed insurance benefits when the child likes providing care. In that case, fixed benefits not only eliminate intra-family moral hazard but also trigger an opposite effect, namely, an increase in the child's caregiving. With proportional benefits, both an increase and a decrease in the child's care provision is possible, which means that intra-family moral hazard cannot be completely ruled out. In addition, even in the case when intra-family moral hazard is not a problem with either type of benefits, it is shown that the child's chosen amount of caregiving is greater with fixed than with proportional insurance benefits. Turning to the parent, it is shown that, in the context of intra-family moral hazard, fixed insurance benefits make insurance more desirable to him. Even though fixed benefits do not always guarantee that the parent will find insurance beneficial, purchasing a positive amount of insurance is more likely to be in the parent's interest when benefits are fixed rather than proportional.

The results of the paper confirm that a low degree of intra-family moral hazard in France could (at least partly) be attributed to the use of fixed insurance benefits. Moreover, the results could also to some extent explain the relative success of the French private LTC insurance market. While the French and the American markets are the world's two most developed markets for private LTC insurance, in 2010, about $15 \%$ of the population aged 40 and over had private LTC insurance in France, compared to only 5\% in the U.S. (Colombo et al., 2011). Given the findings of the paper, it seems reasonable to believe that the use of fixed benefits takes part in the success of France. Indeed, if fixed benefits (through the softening of intra-family moral hazard) can make it more likely that a parent will decide to purchase insurance, the use of this insurance scheme could be seen as a reason for the success of attracting customers in the French LTC insurance market. ${ }^{11}$

While the results in this paper support the idea that fixed benefits help to encourage the demand

\footnotetext{
${ }^{11}$ Fixed benefits have been recognized as contributing to the success of the French market by several authors (Duran and Taleyson (2003), Kessler (2008), Taleyson (2003)). However, these papers do not develop any formal modeling and emphasize somewhat different features of fixed benefit contracts (such as simplicity and flexibility) as the ones that encourage the demand for insurance.
} 
for insurance, it also has to be mentioned that, on the other hand, the use of fixed benefit schemes can be seen as one of the reasons for the non-purchase of LTC insurance. This idea is based on the argument that fixed benefit insurance contracts are incomplete, which might discourage rational agents from purchasing insurance (see Cutler (1993) as well as Cremer et al. (2012) and Pestieau and Ponthière (2011)). However, it should be emphasized that the present paper analyzes the features of insurance benefits focusing exclusively on the context of intra-family moral hazard. Therefore, the paper does not intend to draw general conclusions about the role of the two analyzed types of benefits in LTC insurance but rather aims at exploring them by isolating their effects in one particular context.

The paper is organized as follows. The first section introduces and describes the model. The second section looks at the problem of the child and studies the impact that insurance with fixed and proportional benefits has on her choice of care provision. The third section turns to the parent and analyzes his insurance purchase decisions. Finally, the last section concludes.

\section{The model}

Let us consider an elderly parent $p$ and his adult child $c$. The parent might become dependent (i.e. need LTC) with probability $\pi$ and might remain independent with probability $1-\pi$. Let us discuss the parent and the child in turn.

The expected utility function of the parent can be written as

$$
E U^{p}=\pi U_{D}^{p}+(1-\pi) U_{I}^{p}
$$

where $U_{D}^{p}=u^{p}\left(Q, W_{p}^{D}\right)$ is the parent's utility when he is dependent and $U_{I}^{p}=v^{p}\left(X, W_{p}^{I}\right)$ is his utility when he is independent.

When the parent is not dependent, his utility depends on his consumption level $X$ (with $\frac{\partial v^{p}}{\partial X}>0$ and $\left.\frac{\partial^{2} v^{p}}{(\partial X)^{2}}<0\right)$ and his final wealth $W_{p}^{I}\left(\right.$ with $\frac{\partial v^{p}}{\partial W_{p}^{I}}>0$ and $\left.\frac{\partial^{2} v^{p}}{\left(\partial W_{p}^{I}\right)^{2}}<0\right)$ which will be left at the end of his life as a bequest to the child. ${ }^{12}$ In other words, the parent experiences a joy-of-giving from being able to

\footnotetext{
${ }^{12}$ I assume that the parent does not have any other beneficiaries and thus he will leave all his wealth to the child.
} 
leave a bequest to the child. ${ }^{13}$

When the parent is dependent, similarly to Zweifel and Strüwe (1998), it is assumed for simplicity that he needs a certain exogenously determined total amount of care (time) $\bar{L}$. This amount is fixed and does not enter explicitly his utility function. It can be assumed that if the total amount of care is lower than $\bar{L}$, the parent's utility equals $-\infty$, and if the total amount of care exceeds $\bar{L}$, the utility remains constant. To simplify even more, we can assume that the parent needs care all the time and so $\bar{L}$ measures the total number of hours in the period analyzed. Moreover, it is assumed that a dependent parent's consumption is entirely determined by his disability and neither gives him a particular pleasure nor can be chosen by him. Therefore, a dependent parent's consumption is not included in his utility function and its cost is assumed to be encompassed in the cost of care. However, the parent places a special value on the amount of LTC provided to him by his child $(Q)$. Thus, $Q$ explicitly enters the utility function and it is assumed that $\frac{\partial u^{p}}{\partial Q}>0$ (with $\frac{\partial^{2} u^{p}}{(\partial Q)^{2}}<0$ ). If the child does not provide enough care to reach $\bar{L}$, the parent needs to buy formal care services of the amount $\bar{L}-Q$. Formal care does not give any particular utility to the parent. Its only function is to ensure that $\bar{L}$ is achieved and its amount is thus only reflected in the budget constraint presented below. As in the case of independence, the parent also cares about his final wealth (bequest) $W_{p}^{D}$ (with $\frac{\partial u^{p}}{\partial W_{p}^{D}}>0$ and $\left.\frac{\partial^{2} u^{p}}{\left(\partial W_{p}^{D}\right)^{2}}<0\right){ }^{14}$

The parent can purchase LTC insurance. I consider two different cases in terms of the type of insurance benefits paid. In the first case, the insurance benefit is proportional to the amount of LTC expenditure (that is, the amount of purchased formal care). In other words, in this case insurance directly lowers the price of formal care. This is the type of benefits considered by Zweifel and Strüwe (1998). In the second case, the insurance benefit is fixed and is paid independently of the amount of formal care purchased.

Let us first look at the case of proportional insurance benefits. Denote by $\delta$ the price of one hour of formal provision of LTC and by $\gamma \in[0,1]$ the share of the price that is paid by the insurance company. The amount of formal care that the parent will have to purchase if he becomes dependent will be equal

\footnotetext{
${ }^{13}$ I do not impose a sign on the cross-derivative $\frac{\partial^{2} v^{p}}{\partial X \partial W_{p}^{I}}=\frac{\partial^{2} v^{p}}{\partial W_{p}^{I} \partial X}$, but only assume that $\frac{\partial^{2} v^{p}}{(\partial X)^{2}} \cdot \frac{\partial^{2} v^{p}}{\left(\partial W_{p}^{I}\right)^{2}}-\left[\frac{\partial^{2} v^{p}}{\partial X \partial W_{p}^{I}}\right]^{2}>0$ for all $\left(X, W_{p}^{I}\right)$ so that $v^{p}\left(X, W_{p}^{I}\right)$ is strictly concave.

${ }^{14} \mathrm{I}$ do not impose a sign on the cross-derivative $\frac{\partial^{2} u^{p}}{\partial Q \partial W_{p}^{D}}=\frac{\partial^{2} u^{p}}{\partial W_{p}^{D} \partial Q}$, but it seems likely to be positive since the parent is likely to enjoy leaving a bequest more when the child provides more care to him (even though it could be that the marginal utility of bequest is independent of $Q$ and thus the cross-derivative equal to zero).
} 
to $\bar{L}-Q$. Thus, the (actuarially fair) insurance premium is equal to $\pi \gamma \delta(\bar{L}-Q)$ (the expected benefit from the insurance). ${ }^{15}$ Denoting by $\bar{W}_{p}$ the parent's initial wealth, we can write the parent's final wealth if he is dependent:

$$
W_{p}^{D}=\bar{W}_{p}-\pi \gamma \delta(\bar{L}-Q)-(1-\gamma) \delta(\bar{L}-Q)
$$

and if he is independent:

$$
W_{p}^{I}=\bar{W}_{p}-\pi \gamma \delta(\bar{L}-Q)-X
$$

The parent's choice of insurance is modeled as his choice of $\gamma$.

Now let us turn to the case of fixed insurance benefits. Denoting by $B$ the fixed insurance benefit received in case of dependence, the (actuarially fair) insurance premium is equal to $\pi B$. If the parent is dependent, his final wealth can be written as

$$
W_{p}^{D}=\bar{W}_{p}-\pi B-\delta(\bar{L}-Q)+B
$$

and if he is independent, his final wealth writes as

$$
W_{p}^{I}=\bar{W}_{p}-\pi B-X
$$

Here, the parent's choice of insurance is modeled as his choice of $B$.

Let us now turn to the child. Since we are interested in the child only in the state of nature where the parent becomes dependent, the child's utility is considered only in that case. This utility can be written as

$$
U_{D}^{c}=u^{c}\left(Q, W_{c}^{D}\right)
$$

The child's utility depends on the amount of LTC that she provides to her parent $(Q)$ and on her wealth

\footnotetext{
${ }^{15}$ The insurance company (correctly) anticipates the amount of care provision $Q$ that will be chosen by the child. See also below.
} 
$\left(W_{c}^{D}\right) \cdot{ }^{16}$ As usual, $\frac{\partial u^{c}}{\partial W_{c}^{D}}>0$ and $\frac{\partial^{2} u^{c}}{\left(\partial W_{c}^{D}\right)^{2}}<0$ are assumed. As far as $Q$ is concerned, I will look separately at the case where the child "dislikes" providing care to the parent $\left(\frac{\partial u^{c}}{\partial Q}<0\right)$ and at the case where she "likes" providing care $\left(\frac{\partial u^{c}}{\partial Q}>0\right) .{ }^{17}$ In both cases it is assumed that $\frac{\partial^{2} u^{c}}{(\partial Q)^{2}}<0$, that is, when the child dislikes providing care, her utility (holding $W_{c}^{D}$ constant) is decreasing in $Q$ at an increasing rate, and when the child likes providing care, her utility (holding $W_{c}^{D}$ constant) is increasing in $Q$ at a decreasing rate. $^{18}$

As mentioned above, the parent's final wealth is left to the child as a bequest. Thus, the child's wealth can be written as

$$
W_{c}^{D}=w(\bar{L}-Q)+W_{p}^{D}
$$

where $w$ is the child's hourly wage and $(\bar{L}-Q)$ is the amount of time the child works. ${ }^{19}$

The timing of the model is the following. First, the parent makes the insurance purchase decision. Then, the risk of dependence materializes or not. If the parent remains independent, he chooses his consumption level $X$. If the parent becomes dependent, the child (knowing the insurance decision made by the parent) decides how much LTC she will provide; the rest is purchased by the parent in the form of formal care.

An additional remark could also be made at this point. This concerns the case of proportional insurance benefits. In that case, the insurance premium $\pi \gamma \delta(\bar{L}-Q)$ depends on $Q$. However, when the child makes her choice of $Q$, she considers the premium as fixed because at that point the premium is already paid and cannot be changed. The child chooses $Q$ which depends on the insurance coverage reflected by $\gamma$. By backward induction, the insurance company (correctly) anticipates $Q(\gamma)$ and bases the premium on it: $\pi \gamma \delta(\bar{L}-Q(\gamma))$. In the same way, the parent also (correctly) anticipates $Q(\gamma)$ and

\footnotetext{
${ }^{16}$ Since we are interested in the child's choice of care for the parent and not in her consumption decisions, to simplify the presentation I assume that the child's utility depends only on her wealth and not on her wealth and consumption.

${ }^{17}$ In general, caregiving might at the same time be associated both with a certain degree of disutility and with a certain degree of utility coming, for instance, from altruistic feelings or the appreciation of the time spent with the parent. Thus, the cases "the child dislikes providing care" and "the child likes providing care" can be seen as shortcuts to reflect respectively the situation when the disutility of caregiving offsets the utility and the situation when the utility of caregiving offsets the disutility.

${ }^{18} \mathrm{I}$ do not impose a sign on the cross-derivative $\frac{\partial^{2} u^{c}}{\partial Q \partial W_{c}^{D}}=\frac{\partial^{2} u^{c}}{\partial W_{c}^{D} \partial Q}$, but only assume $\frac{\partial^{2} u^{c}}{(\partial Q)^{2}} \cdot \frac{\partial^{2} u^{c}}{\left(\partial W_{c}^{D}\right)^{2}}-\left[\frac{\partial^{2} u^{c}}{\partial Q \partial W_{c}^{D}}\right]^{2}>0$ for all $\left(Q, W_{c}^{D}\right)$ so that $u^{c}\left(Q, W_{c}^{D}\right)$ is strictly concave.

${ }^{19}$ As noted above, I assume that $\bar{L}$ measures the total number of hours in the period analyzed.
} 
takes it into account in his expected utility maximization.

We proceed by reasoning backwards and start by looking at the caregiving choice of the child.

\section{Fixed vs Proportional: The impact on the child's choice}

This section looks at the child's choice of LTC provision in the state of nature when the parent becomes dependent. In particular, the aim of the analysis is to compare the impact that insurance coverage has on the child's caregiving choice with proportional and with fixed insurance benefits. As mentioned above,

I will consider separately the case where the child dislikes providing care to the parent $\left(\frac{\partial u^{c}}{\partial Q}<0\right)$ and the case where she likes providing care $\left(\frac{\partial u^{c}}{\partial Q}>0\right)$.

\section{Case 1: the child dislikes providing care $\left(\frac{\partial u^{c}}{\partial Q}<0\right)$}

First, it should be noted that at this stage of the analysis the parent's choice of insurance is already made; thus, the value of $\gamma$ or $B$ is considered by the child as given. Having this in mind, we can characterize three possible ranges for the values of the child's wage $w$ :

- $w \geq \delta$, that is, the child's wage is higher than the (full) price of formal care. In this case, the child will never decide to provide care to the parent, even if the parent has no insurance coverage. This is because care provision gives no benefit to the child: on the one hand, she dislikes providing care; on the other hand, by providing care she gains less in terms of the parent's bequest than loses by not participating in the labour market. This case is not interesting for comparing the impact of different insurance benefits and thus will not be considered in what follows.

- $(1-\gamma) \delta \leq w<\delta$, that is, the child's wage is lower than the full price of formal care but higher than the price of formal care when insurance with proportional benefits has been purchased. Here, we will again have a corner solution $Q=0$ in the case of insurance with proportional benefits. However, in the case of fixed benefits, the full price of formal care has to be considered, and thus, in that case, there will be an interior solution for $Q$. In what follows, I will focus only on interior solutions; therefore, due to the corner solution with proportional benefits, this range for $w$ will not 
be considered. On the other hand, here it is quite clear that the child will provide more care in the case of fixed benefits than in the case of proportional ones.

- $w<(1-\gamma) \delta$, that is, the child's wage is lower even than the price of formal care when insurance with proportional benefits has been purchased. In this case, we have interior solutions with both types of insurance benefits. I will thus further focus on this range for $w$.

The child's problem in the case of proportional insurance benefits can be written as follows:

$$
\begin{gathered}
\max _{Q, W_{c}^{D}} U_{D}^{c}=u^{c}\left(Q, W_{c}^{D}\right) \\
\text { s.t. } \quad W_{c}^{D}=w(\bar{L}-Q)+\bar{W}_{p}-\pi \gamma \delta(\bar{L}-Q)-(1-\gamma) \delta(\bar{L}-Q) \\
\Longleftrightarrow \\
W_{c}^{D}-[(1-\gamma) \delta-w] Q=\bar{W}_{p}-\pi \gamma \delta(\bar{L}-Q)-[(1-\gamma) \delta-w] \bar{L}
\end{gathered}
$$

Written in this way - in particular, with the budget constraint rearranged in the form of equation (10) - the child's problem reminds a standard consumer's problem with commodities $Q$ and $W_{c}^{D}$, where commodity $Q$ is a "bad". Since $(1-\gamma) \delta>w$, the term $[(1-\gamma) \delta-w]$ is positive and measures how much money the child saves by providing one additional hour of care (i.e. it is the difference between the price of formal care that is not paid (and thus does not decrease the parent's bequest) and the child's wage which could have been earned instead of providing care). Denoting this term by $p_{p r}$, we have the following budget constraint:

$$
W_{c}^{D}-p_{p r} Q=\underbrace{\bar{W}_{p}-\pi \gamma \delta(\bar{L}-Q)-p_{p r} \bar{L}}_{I_{p r}}
$$

At the point when the child makes the decision, the insurance premium $\pi \gamma \delta(\bar{L}-Q)$ is already paid by the parent and is thus considered as fixed by the child. Therefore, the right-hand side of (11) can be seen as the exogenous income of the child (denoted by $I_{p r}$ ), whereas the left-hand side can be seen as the child's expenditure. Commodity $Q$ has a negative "price" $-p_{p r}$, while the price of $W_{c}^{D}$ is $1 . Q$ is thus a 
bad in terms of utility, but it gives a financial gain to the child.

In a similar way, the child's budget constraint in the case of fixed insurance benefits can be written as

$$
W_{c}^{D}-p_{f} Q=\underbrace{\bar{W}_{p}-\pi B+B-p_{f} \bar{L}}_{I_{f}}
$$

where $p_{f}=\delta-w>p_{p r}$. Thus, with fixed benefits the child's hourly gain from providing care is higher.

Solving the above defined child's problem would give the child's chosen amounts of $W_{c}^{D}$ and $Q$, which would be functions of $p_{p r}$ and $I_{p r}$ or $p_{f}$ and $I_{f}$. Following the standard consumer's theory, we can derive the Slutsky equation for the child's supply of care:

$$
\frac{\partial Q}{\partial p_{i}}=\frac{\partial \tilde{Q}}{\partial p_{i}}+Q \frac{\partial Q}{\partial I_{i}} \quad i=p r, f
$$

where $\tilde{Q}$ is the compensated supply of care. For further use, let us discuss the signs of the two terms. The compensated term $\frac{\partial \tilde{Q}}{\partial p_{i}}$ is positive because $p_{i}$ measures the child's gain from providing one additional hour of care. Since care provision is a bad for the child $\left(\frac{\partial u^{c}}{\partial Q}<0\right)$, the time left after providing care $(\bar{L}-Q)$ increases her utility. If we assume that this time of "leisure" from providing care is a normal good for the child, then we have $\frac{\partial Q}{\partial I_{i}}<0$.

We can now turn to our question of interest, that is, the effect of insurance on $Q$. In the case of proportional insurance benefits we have:

$$
\frac{\partial Q\left(p_{p r}, I_{p r}\right)}{\partial \gamma}=\frac{\partial Q}{\partial p_{p r}} \frac{\partial p_{p r}}{\partial \gamma}+\frac{\partial Q}{\partial I_{p r}} \frac{\partial I_{p r}}{\partial \gamma}=-\delta\left[\frac{\partial \tilde{Q}}{\partial p_{p r}}+Q \frac{\partial Q}{\partial I_{p r}}\right]+\frac{\partial Q}{\partial I_{p r}}\left[-\pi \delta(\bar{L}-Q)+\pi \gamma \delta \frac{\partial Q}{\partial \gamma}+\delta \bar{L}\right]
$$




$$
\frac{\partial Q}{\partial \gamma}=\frac{\overbrace{-\delta \frac{\partial \tilde{Q}}{\partial p_{p r}}}^{(-)}+\overbrace{Q \frac{\partial Q}{\partial I_{p r}}(-\delta)}^{(+)}+\overbrace{\frac{\partial Q}{\partial I_{p r}}[\delta \bar{L}(1-\pi)+\pi \delta Q]}^{(-)}}{\underbrace{1-\frac{\partial Q}{\partial I_{p r}} \pi \gamma \delta}_{(+)}}
$$

We should first determine the sign of $\frac{\partial Q}{\partial \gamma}$. Since the denominator of equation (15) is positive, the sign of $\frac{\partial Q}{\partial \gamma}$ depends on the numerator. Let us thus discuss the terms in the numerator. The first term in the numerator is negative: since insurance reduces the price of formal care, the child saves less by providing one additional hour of informal caregiving and this decrease in her hourly gain pushes her to provide less care. This is the substitution effect. The second term is the income effect coming from the change in the hourly gain. It is positive: the decrease in the hourly gain means that the child is now saving less money in total (she is "poorer"), so she wants to provide more care to increase her total savings. However, there is also an additional income effect which is reflected by the third term. It comes from the fact that the child's exogenous income $\left(I_{p r}\right)$ depends on the amount of insurance as well. The third term in the numerator is negative: insurance increases the exogenous income of the child and thus the child can afford reducing care provision which is a bad for her (equivalently, she can afford increasing her free time from caregiving). If we combine the two income effects, we get the following:

$$
\frac{\partial Q}{\partial \gamma}=\frac{\overbrace{\delta \frac{\partial \tilde{Q}}{\partial p_{p r}}}^{(-)}+\overbrace{\frac{\partial Q}{\partial I_{p r}} \delta(1-\pi)(\bar{L}-Q)}^{(-)}}{\underbrace{1-\frac{\partial Q}{\partial I_{p r}} \pi \gamma \delta}_{(+)}}<0
$$

Thus, due to the additional income effect, the total income effect is negative. Together with the negative substitution effect, this implies that the child's caregiving is decreasing in the insurance coverage that the parent has. 
Now let us look at the case of fixed insurance benefits:

$$
\frac{\partial Q\left(p_{f}, I_{f}\right)}{\partial B}=\frac{\partial Q}{\partial p_{f}} \underbrace{\frac{\partial p_{f}}{\partial B}}_{=0}+\frac{\partial Q}{\partial I_{f}} \frac{\partial I_{f}}{\partial B}=\underbrace{\frac{\partial Q}{\partial I_{f}}}_{(-)}(1-\pi)<0
$$

In this case, insurance coverage does not affect the hourly gain from care provision but only has an impact on the child's exogenous income. As in the case of proportional benefits, the child's exogenous income is increased, which implies that she can afford reducing her caregiving.

The above results can be summarized as the following proposition:

Proposition 1. Consider a child who dislikes providing LTC to her parent and assume that $w<$ $(1-\gamma) \delta$ holds after the parent's purchase of insurance. The amount of the child's caregiving is decreasing in the parent's insurance coverage both in the case of proportional and in the case of fixed insurance benefits.

Thus, insurance induces the child to reduce her care provision in both cases of insurance benefits. However, it is important to compare the magnitudes of the reduction. To do this, we first have to make things comparable. In particular, if in the fixed benefits case the insurance benefit $B$ is increased by 1 euro, in the proportional benefits case we should also look at the situation where the insurance benefit is increased by 1 euro, i.e. $d[\gamma \delta(\bar{L}-Q)]=1$. In other words, we have to compare (17) to

$$
\left.\frac{\partial Q}{\partial \gamma} \cdot d \gamma\right|_{d[\gamma \delta(\bar{L}-Q)]=1}=\frac{-\delta \frac{\partial \tilde{Q}}{\partial p_{p r}}+\frac{\partial Q}{\partial I_{p r}} \delta(1-\pi)(\bar{L}-Q)}{1-\frac{\partial Q}{\partial I_{p r}} \pi \gamma \delta} \cdot \frac{1}{\delta(\bar{L}-Q)-\gamma \delta \frac{\partial Q}{\partial \gamma}}
$$

To compare these two expressions, we need to take a common starting point. A natural starting point seems to be the situation when there is no insurance coverage. Evaluating $\left.\frac{\partial Q}{\partial \gamma} \cdot d \gamma\right|_{d[\gamma \delta(\bar{L}-Q)]=1}$ and $\frac{\partial Q}{\partial B}$ at the point with no insurance, we get the following:

$$
\left.\left(\left.\frac{\partial Q}{\partial \gamma} \cdot d \gamma\right|_{d[\gamma \delta(\bar{L}-Q)]=1}\right)\right|_{\gamma=0}=-\left.\frac{1}{(\bar{L}-Q)} \cdot \frac{\partial \tilde{Q}}{\partial p_{p r}}\right|_{\gamma=0}+\left.\frac{\partial Q}{\partial I_{p r}}\right|_{\gamma=0}(1-\pi)
$$

and

$$
\left.\frac{\partial Q}{\partial B}\right|_{B=0}=\left.\frac{\partial Q}{\partial I_{f}}\right|_{B=0}(1-\pi)
$$


The two expressions are negative, but, since $\left.\frac{\partial Q}{\partial I_{p r}}\right|_{\gamma=0}=\left.\frac{\partial Q}{\partial I_{f}}\right|_{B=0}($ and $<0)$, it can be easily seen that the expression in (19) is larger in absolute value than the one in (20) because it has an additional term $-\left.\frac{1}{(\bar{L}-Q)} \cdot \frac{\partial \tilde{Q}}{\partial p_{p r}}\right|_{\gamma=0}<0$. Thus, the purchase of 1 euro of insurance coverage induces the child to reduce the provision of care more when insurance benefits are proportional than when they are fixed. We can therefore formulate the following proposition:

Proposition 2. Consider a child who dislikes providing LTC to her parent and assume that $w<$ $(1-\gamma) \delta$ holds after the parent's purchase of insurance. The parent's purchase of the first euro of insurance coverage induces the child to decrease the amount of her caregiving (with respect to the amount that she would provide if the parent had no insurance) more when insurance benefits are proportional than when they are fixed.

This proposition provides preliminary support for the idea that fixed insurance benefits help to mitigate intra-family moral hazard. On the other hand, it only concerns the marginal effect of insurance with respect to the situation of zero insurance coverage. A more general result can nevertheless be derived as well. It is stated in Proposition 3:

Proposition 3. Consider a child who dislikes providing LTC to her parent and assume that $w<$ $(1-\gamma) \delta$ holds after the parent's purchase of insurance. When the amount of benefits obtained by the parent from both types of insurance is the same, the child chooses to provide more care in the case of fixed benefits than in the case of proportional benefits. In addition, the child is better-off in the case of fixed benefits.

Proposition 3 is proved graphically in Figure 1 below. Figure 1 depicts the child's preferences in terms of her wealth and the time left after providing care to the parent $(\bar{L}-Q)$. Since care provision is a bad for the child $\left(\frac{\partial u^{c}}{\partial Q}<0\right)$, this "free" time increases her utility. Therefore, the child's preferences can be depicted using "standard" indifference curves. ${ }^{20}$

Line NT depicts the child's budget constraint in the case where the parent has purchased insurance

20 More precisely, we need to define $\mu^{c}\left(\bar{L}-Q, W_{c}^{D}\right)=u^{c}\left(\bar{L}-(\bar{L}-Q), W_{c}^{D}\right)=u^{c}\left(Q, W_{c}^{D}\right)$. Note that $\frac{\partial \mu^{c}}{\partial(L-Q)}=$ $-\frac{\partial u^{c}}{\partial Q}>0, \frac{\partial^{2} \mu^{c}}{(\partial(\bar{L}-Q))^{2}}=\frac{\partial^{2} u^{c}}{(\partial Q)^{2}}<0, \frac{\partial \mu^{c}}{\partial W_{c}^{D}}=\frac{\partial u^{c}}{\partial W_{c}^{D}}>0, \frac{\partial^{2} \mu^{c}}{\left(\partial W_{c}^{D}\right)^{2}}=\frac{\partial^{2} u^{c}}{\left(\partial W_{c}^{D}\right)^{2}}<0$ and $\frac{\partial^{2} \mu^{c}}{\partial(\bar{L}-Q) \partial W_{c}^{D}}=-\frac{\partial^{2} u^{c}}{\partial Q \partial W_{c}^{D}}$, which implies that $\mu^{c}\left(\bar{L}-Q, W_{c}^{D}\right)$ is also strictly concave (see footnote 18) and thus the indifference curves are strictly convex as depicted in Figure 1. 
with proportional benefits (rearranging equation (9) it can be seen that it has a slope of $-[(1-\gamma) \delta-w]$ ). Given this budget constraint, the child's optimal amount of "free" time is $(\bar{L}-Q)^{p r}$ (since the insurance company anticipates the child's choice, the insurance premium that was paid by the parent was based on this amount: $\left.\pi \gamma \delta(\bar{L}-Q)^{p r}\right)$.

To see graphically the amount of insurance benefits obtained in the proportional case, we can make use of line NM. Line NM depicts a hypothetical budget constraint that the child would have if the parent had paid the insurance premium $\pi \gamma \delta(\bar{L}-Q)^{p r}$ but had not received any insurance benefits (thus, the slope of NM is $-[\delta-w]$ ). Therefore, the difference E'F shows the amount of insurance benefits obtained (this amount is equal to $\left.\gamma \delta(\bar{L}-Q)^{p r}\right)$.

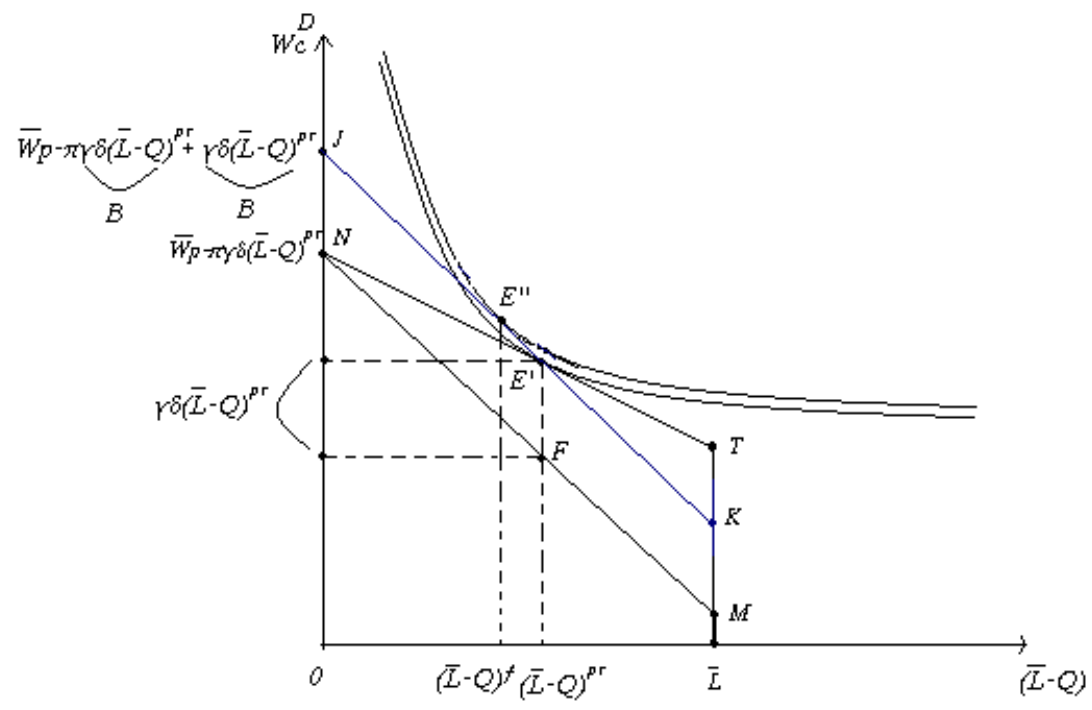

Figure 1. Choices of a child who dislikes providing care

Now let us consider insurance with fixed benefits where the amount of benefits $B$ is equal to the amount obtained in the proportional case, i.e. $B=\gamma \delta(\bar{L}-Q)^{p r}$. In the case where the parent has purchased such insurance, the child's budget constraint can be represented by line JK (which is parallel to NM and thus has a slope of $-[\delta-w])$. In this case the equilibrium is at E", and the amount of "free" 
time chosen by the child is $(\bar{L}-Q)^{f}<(\bar{L}-Q)^{p r}$. Thus, the child chooses less "free" time, which means that she provides more care to the parent. Also, it should be noted that at E" the child reaches a higher indifference curve and thus has a higher level of utility. This proves Proposition 3.

To sum up, we have just seen, in the case of a child who dislikes providing care, that even though the child's caregiving is decreasing in insurance coverage with both types of benefits, the same amount of LTC insurance purchased by the parent results in more care given by the child when insurance benefits are fixed than when they are proportional. This indeed confirms that the phenomenon of intra-family moral hazard, although still existent, is less severe with fixed insurance benefits.

\section{Case 2: the child likes providing care $\left(\frac{\partial u^{c}}{\partial Q}>0\right)$}

As in Case 1, let us start by defining the interval for the child's wage $w$ that we will focus on. There are again three possibilities:

- $w \leq(1-\gamma) \delta$. In this case, the child will provide care all the time $(Q=\bar{L})$ irrespective of the parent's insurance coverage. Thus, this case is not interesting for our analysis and will not be considered further.

- $(1-\gamma) \delta<w \leq \delta$. Here, we will again have the corner solution $Q=\bar{L}$ in the case of fixed insurance benefits, whereas with proportional insurance benefits there will be an interior solution. As before, this intermediate case will not be considered since it involves a corner solution; however, it again seems clear that with proportional benefits the child will provide less care than with fixed ones.

- $w>\delta$. In this case, we have interior solutions with both types of insurance benefits. Thus, the further analysis will be focused on this range for $w$.

The problem of the child writes in the same way as in Case 1, but the important difference is that commodity $Q$ is no longer a bad for the child (the child now enjoys providing care). Moreover, it is now more convenient to slightly rearrange the child's budget constraint. In particular, in the case of proportional insurance benefits the budget constraint can be written as

$$
W_{c}^{D}+[w-(1-\gamma) \delta] Q=\bar{W}_{p}-\pi \gamma \delta(\bar{L}-Q)+[w-(1-\gamma) \delta] \bar{L}
$$


Since $w>(1-\gamma) \delta$, the term $[w-(1-\gamma) \delta]$ is positive and measures the opportunity cost of providing one additional hour of care. Denoting this term by $\rho_{p r}$, we have

$$
W_{c}^{D}+\rho_{p r} Q=\underbrace{\bar{W}_{p}-\pi \gamma \delta(\bar{L}-Q)+\rho_{p r} \bar{L}}_{M_{p r}}
$$

As in Case 1, the right-hand side of (22) can be seen as the exogenous income of the child (here denoted by $M_{p r}$ ), whereas the left-hand side can be seen as the child's expenditure. Commodity $Q$ now has a positive price $\rho_{p r}$. In other words, the child enjoys helping the parent, but this has a cost because by not working on the labour market she forgoes more than is saved by reducing formal care.

In a similar way, the child's budget constraint in the case of fixed benefits can be written as

$$
W_{c}^{D}+\rho_{f} Q=\underbrace{\bar{W}_{p}-\pi B+B+\rho_{f} \bar{L}}_{M_{f}}
$$

where $\rho_{f}=w-\delta<\rho_{p r}$. Thus, the opportunity cost of providing one additional hour of care is lower with fixed insurance benefits.

Similarly to Case 1, solving the above defined child's problem would give the child's chosen amounts of $W_{c}^{D}$ and $Q$, which would be functions of $\rho_{p r}$ and $M_{p r}$ or $\rho_{f}$ and $M_{f}$. We can again derive the Slutsky equation for the child's supply of care, which now writes as follows:

$$
\frac{\partial Q}{\partial \rho_{i}}=\frac{\partial \tilde{Q}}{\partial \rho_{i}}-Q \frac{\partial Q}{\partial M_{i}} \quad i=p r, f
$$

The compensated term $\frac{\partial \tilde{Q}}{\partial \rho_{i}}$ is negative since $\rho_{i}$ measures the child's opportunity cost. If care provision is a normal good for the child, then we have $\frac{\partial Q}{\partial M_{i}}>0$.

We can now examine the effect of insurance on $Q$. Proceeding as in Case 1, with proportional insurance benefits we obtain 


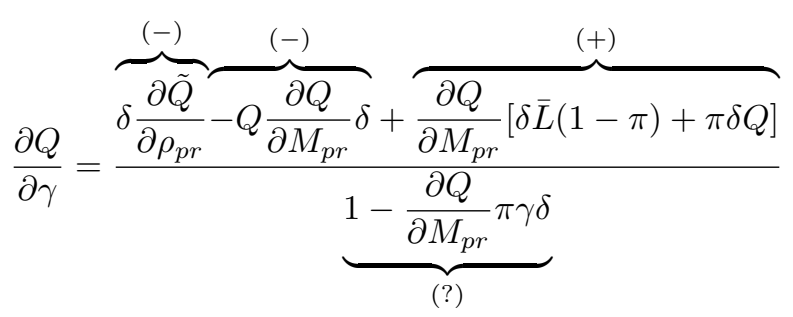

To determine the sign of $\frac{\partial Q}{\partial \gamma}$, let us start with the numerator of (25). The first term in the numerator is negative: since insurance decreases the price of formal care, the child's opportunity cost is increased and this pushes her to provide less care. This is the substitution effect. The second term is the income effect coming from the change in the opportunity cost. It is also negative: the increase in the opportunity cost means that the child is now losing more money in total by providing care to the parent, so she wants to provide less care since she is now "poorer" and care provision is a normal good. However, there is again an additional income effect (the third term in the numerator). It again comes from the fact that the child's exogenous income $\left(M_{p r}\right)$ depends on the amount of insurance as well. As in Case 1, insurance increases the exogenous income of the child, but now the effect on caregiving is different since $Q$ is no longer a bad. Since caregiving is enjoyable but costly, the increase in the exogenous income induces the child to provide more care as she is now wealthier and can afford spending more time on the activity that she enjoys. The third term in the numerator is thus positive. If we combine the two income effects, we get the following:

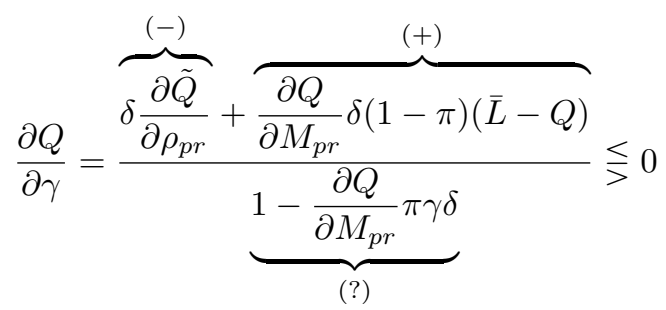

Due to the additional income effect, the total income effect is positive. However, the substitution effect is negative and so the sign of the total effect in the numerator is not clear. In addition, the sign of the denominator is ambiguous as well. Therefore, the effect of insurance with proportional benefits on the child's caregiving is not clear in this case. It might be that insurance will decrease the child's care 
provision, but it might also happen that, on the contrary, the child will be induced to provide more care.

Turning to the case of fixed insurance benefits, we obtain the following:

$$
\frac{\partial Q}{\partial B}=\underbrace{\frac{\partial Q}{\partial M_{f}}}_{(+)}(1-\pi)>0
$$

As in Case 1, with fixed benefits, insurance only has an impact on the child's exogenous income, which is increased. However, since caregiving is now enjoyable to the child, the increase in the exogenous income fosters care provision, just as explained in the discussion of proportional benefits. As the exogenous income effect is the only effect present when benefits are fixed, insurance coverage unambiguously increases the child's caregiving. In other words, when the child likes providing care, insurance with fixed benefits clearly does not cause intra-family moral hazard and even triggers an opposite effect, namely, a rise in care provision..$^{21}$

We can summarize the above results as follows:

Proposition 4. Consider a child who likes providing LTC to her parent and has a wage $w>\delta$. The amount of the child's caregiving is increasing in the parent's insurance coverage in the case of fixed insurance benefits. In the case of proportional benefits, the effect of insurance on the child's caregiving is ambiguous.

The fact that intra-family moral hazard disappears with fixed benefit insurance but might still be present with proportional benefits (since a reduction in the child's caregiving cannot be ruled out in that case) can be seen as an advantage of fixed benefit contracts. However, since the case when the child's caregiving is increased with both types of benefits is also possible, a deeper analysis should be made.

First, as in Case 1, we can compare the child's caregiving responses to the purchase of 1 euro of insurance evaluated at the point of zero coverage. In particular, we can look at

\footnotetext{
${ }^{21}$ In general, an increase in the child's caregiving could be seen as a form of moral hazard as well since it is also a modification of the child's behavior caused by the presence of insurance. However, following the original argument of Pauly (1990), I define intra-family moral hazard as a reduction in the child's care provision due to insurance coverage and thus refer to an increase in the child's caregiving as to an effect opposite to intra-family moral hazard.
} 


$$
\left.\left(\left.\frac{\partial Q}{\partial \gamma} \cdot d \gamma\right|_{d[\gamma \delta(\bar{L}-Q)]=1}\right)\right|_{\gamma=0}=\frac{1}{(\bar{L}-Q)} \cdot \underbrace{\left.\frac{\partial \tilde{Q}}{\partial \rho_{p r}}\right|_{\gamma=0}}_{(-)}+\underbrace{\left.\frac{\partial Q}{\partial M_{p r}}\right|_{\gamma=0}}_{(+)}(1-\pi)
$$

and

$$
\left.\frac{\partial Q}{\partial B}\right|_{B=0}=\underbrace{\left.\frac{\partial Q}{\partial M_{f}}\right|_{B=0}}_{(+)}(1-\pi)
$$

It is already clear from Proposition 4 and also from equation (29) that with fixed benefits the child's caregiving is increased. With proportional benefits, we can see that if

$$
-\left.\frac{1}{(\bar{L}-Q)} \cdot \frac{\partial \tilde{Q}}{\partial \rho_{p r}}\right|_{\gamma=0}>\left.(\text { resp. }=) \frac{\partial Q}{\partial M_{p r}}\right|_{\gamma=0}(1-\pi)
$$

then the child reduces (resp. does not change) her caregiving. The child's caregiving is increased only if $-\left.\frac{1}{(\bar{L}-Q)} \cdot \frac{\partial \tilde{Q}}{\partial \rho_{p r}}\right|_{\gamma=0}<\left.\frac{\partial Q}{\partial M_{p r}}\right|_{\gamma=0}(1-\pi)$. However, we can see that the increase is smaller than with fixed benefits since $\left.\frac{\partial Q}{\partial M_{p r}}\right|_{\gamma=0}=\left.\frac{\partial Q}{\partial M_{f}}\right|_{B=0}$ and the first term in (28) is negative. This can be summarized in the following proposition:

Proposition 5. Consider a child who likes providing LTC to her parent and has a wage $w>\delta$. If with proportional benefits the parent's purchase of the first euro of insurance coverage induces the child to increase the amount of her caregiving (with respect to the amount that she would provide if the parent had no insurance), this increase is smaller than the increase in the case of fixed benefits.

Proposition 5 provides some preliminary evidence that, similarly to Case 1, insurance with fixed benefits is associated with a higher amount of the child's care provision in this case as well. The analysis below will confirm that this is indeed true. In particular, the analysis below will prove the following proposition (which is a counterpart here of Proposition 3 in Case 1):

Proposition 6. Consider a child who likes providing LTC to her parent and has a wage $w>\delta$. When the amount of benefits obtained by the parent from both types of insurance is the same, the child chooses to provide more care in the case of fixed benefits than in the case of proportional benefits. In addition, the child is better-off in the case of fixed benefits.

Similarly to Proposition 3, Proposition 6 is proved graphically. The analysis is based on Figure 2 
which depicts the child's preferences in terms of her wealth and care provision to the parent.

Line JT depicts the child's budget constraint in the case where the parent has purchased insurance with proportional benefits (it can be represented by equation (21) above and has a slope of $-[w-(1-\gamma) \delta]$ ). Given this budget constraint, the child's optimal amount of care provision is $Q^{p r}$.

Line NT depicts a hypothetical budget constraint that the child would have if the parent had paid the insurance premium $\pi \gamma \delta\left(\bar{L}-Q^{p r}\right)$ but had not received any insurance benefits (the slope of NT is $-[w-\delta])$. The difference E'F shows the amount of insurance benefits obtained in the proportional case (this amount is equal to $\gamma \delta\left(\bar{L}-Q^{p r}\right)$ ).

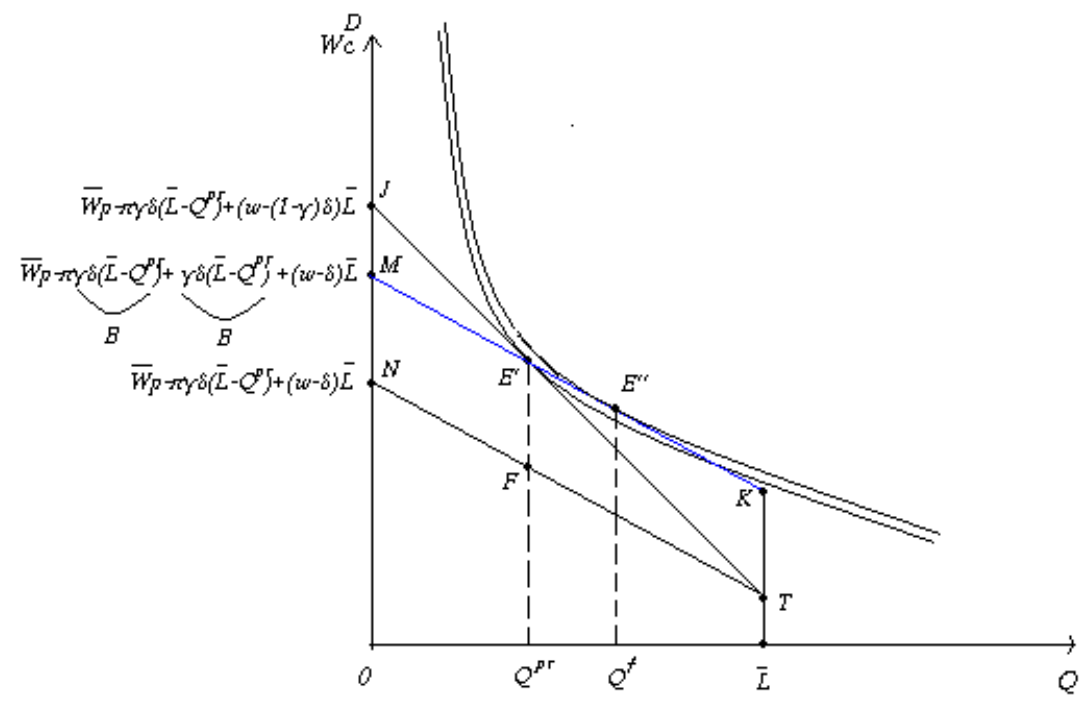

Figure 2. Choices of a child who likes providing care

Now let us consider insurance with fixed benefits where the amount of benefits is $B=\gamma \delta\left(\bar{L}-Q^{p r}\right)$. In the case where the parent has purchased such insurance, the child's budget constraint can be represented by line MK (parallel to NT). In this case the equilibrium is at E", and the amount of care provision chosen by the child is $Q^{f}>Q^{p r}$. Thus, the child chooses to provide more care to the parent. Also, at E" the child reaches a higher indifference curve. This proves Proposition 6 .

To summarize, we have seen that when the child likes providing care, it is possible not to have intra- 
family moral hazard at all. This phenomenon will never be present in the case of fixed insurance benefits, whereas it might still arise with proportional ones. Thus, while in Case 1 fixed insurance benefits play the role of mitigating intra-family moral hazard, here they not only eliminate this phenomenon but also trigger an opposite effect, namely, an increase in the child's caregiving. It is true that an increase in the child's care provision is possible with proportional benefits as well; however, in any event, the child's chosen amount of caregiving is always greater with fixed insurance benefits. Therefore, even in the case when intra-family moral hazard is not a problem, fixed insurance benefits still allow the parent to enjoy more care given by his child.

\section{Fixed vs Proportional: The impact on the parent's insurance decision}

Having compared the impact of insurance on the child's care provision in the cases of fixed and proportional benefits, we can now see how this affects the parent's decision of whether to purchase LTC insurance or not. More specifically, the aim of the analysis is to explore with which type of insurance benefits the parent is more likely to decide to buy a positive amount of insurance. As before, let us discuss separately the two cases of the child's caregiving preferences.

\section{Case 1: the child dislikes providing care $\left(\frac{\partial u^{c}}{\partial Q}<0\right)$}

As in the analysis of the child, in this case I am going to focus on relatively low levels of the child's wage. Note that if $w \geq \delta$, the parent will be indifferent between the two types of insurance benefits since the child will never provide care anyway, which is not interesting for our study.

To examine the parent's decision of whether to purchase insurance or not, we have to look at his expected utility maximization problem. Recall that the parent's expected utility is given by equation (1). The parent has to choose the amount of insurance knowing that it will have an effect on his wealth (bequest) in both states of nature, on his consumption level in the state of independence and on the child's chosen amount of caregiving in the state of dependence. 
It should first be noted that if the parent remains independent, he chooses his consumption level by maximizing $v^{p}\left(X, W_{p}^{I}\right)$ with respect to $X$, which gives the following FOC:

$$
\frac{\partial v^{p}}{\partial X}-\frac{\partial v^{p}}{\partial W_{p}^{I}}=0
$$

To determine whether it is optimal or not for the parent to purchase a positive amount of insurance, we have to study the sign of the derivative of his expected utility function evaluated at the point of zero insurance. Deriving with respect to insurance, making use of equation (30) and evaluating at zero coverage, respectively in the cases of proportional and fixed insurance benefits we get:

$$
\left.\frac{\partial E U^{p}}{\partial \gamma}\right|_{\gamma=0}=\underbrace{\pi(1-\pi) \delta(\bar{L}-Q)}_{(+)} \underbrace{\left(\left.\frac{\partial u^{p}}{\partial W_{p}^{D}}\right|_{\gamma=0}-\left.\frac{\partial v^{p}}{\partial W_{p}^{I}}\right|_{\gamma=0}\right)}_{(+)}+\pi \underbrace{\left.\frac{\partial Q}{\partial \gamma}\right|_{\gamma=0}}_{(-)} \underbrace{\left(\left.\frac{\partial u^{p}}{\partial Q}\right|_{\gamma=0}+\left.\delta \frac{\partial u^{p}}{\partial W_{p}^{D}}\right|_{\gamma=0}\right)}_{(+)}
$$

and

$$
\left.\frac{\partial E U^{p}}{\partial B}\right|_{B=0}=\underbrace{\pi(1-\pi)}_{(+)} \underbrace{\left(\left.\frac{\partial u^{p}}{\partial W_{p}^{D}}\right|_{B=0}-\left.\frac{\partial v^{p}}{\partial W_{p}^{I}}\right|_{B=0}\right)}_{(+)}+\pi \underbrace{\left.\pi \frac{\partial Q}{\partial B}\right|_{B=0}}_{(-)} \underbrace{\left(\left.\frac{\partial u^{p}}{\partial Q}\right|_{B=0}+\left.\delta \frac{\partial u^{p}}{\partial W_{p}^{D}}\right|_{B=0}\right)}_{(+)}
$$

For the case of proportional benefits, a note should be made that here we look at a marginal increase of $\gamma$ in the neighbourhood of $\gamma=0$; thus, recalling that we are interested in $w<\delta$, we can reasonably assume to be in the case $w<(1-\gamma) \delta$ which ensures interior solutions.

Both in the case of proportional and in the case of fixed benefits the derivative has two terms. The second term appears due to the effect of insurance on the child's caregiving $Q$. The parent derives utility from the child's caregiving directly and also through the fact that it reduces the expenditure on formal care. Thus, since $Q$ is decreasing in insurance coverage (Proposition 1), the second term in the derivative is negative and goes against the purchase of insurance. If insurance did not have any effect on $Q$, the second term would be equal to zero and only the first term would be left. 
The first term reflects the parent's motive to insure his bequest. It should be noted that it is not guaranteed that this term will always be positive. In particular, the comparison of $\frac{\partial u^{p}}{\partial W_{p}^{D}}$ and $\frac{\partial v^{p}}{\partial W_{p}^{I}}$ at the point of zero insurance coverage depends on the level of the parent's final wealth with no insurance in each state of nature. If the level of the child's care provision when there is no insurance is sufficiently high, it might be that the expenditure on formal care in the state of dependence is smaller than the parent's consumption in the state of independence, which implies that $W_{p}^{D}$ might be higher than $W_{p}^{I}$. In such a case, it is likely that $\frac{\partial u^{p}}{\partial W_{p}^{D}}<\frac{\partial v^{p}}{\partial W_{p}^{I}}$ (even though it still depends on the exact forms of the two marginal utility functions which are allowed to be different). If that is the case, the parent will not buy insurance neither with proportional nor with fixed benefits (and would not do so even if insurance did not affect $Q$ ), which is not interesting for our analysis. Therefore, I focus on situations when the first term of the derivative is positive (i.e. $\left.\left.\frac{\partial u^{p}}{\partial W_{p}^{D}}\right|_{\gamma=0}\left(=\left.\frac{\partial u^{p}}{\partial W_{p}^{D}}\right|_{B=0}\right)-\left.\frac{\partial v^{p}}{\partial W_{p}^{T}}\right|_{\gamma=0}\left(=\left.\frac{\partial v^{p}}{\partial W_{p}^{T}}\right|_{B=0}\right)>0\right)$. In other words, I look at the cases when the parent would definitely buy some insurance if it had no impact on $Q$ but might decide not to do so when the effect on $Q$ is taken into account. Such situations will occur if with no insurance the parent's expenditure on formal care is relatively high compared to his consumption level in the state of independence. Moreover, the occurrence of such situations becomes more likely if the parent has a higher marginal utility of bequest when he is dependent (that is, if $\frac{\partial u^{p}}{\partial W_{p}^{D}}>\frac{\partial v^{p}}{\partial W_{p}^{T}}$ for $\left.W_{p}^{D}=W_{p}^{I}\right)$, which seems to be quite reasonable if we think that the parent might be more willing to "award" the child in the state of nature where he needs her help.

As mentioned above, the aim of the analysis is to explore in which case - with fixed or with proportional benefits - the parent is more likely to decide to purchase a positive amount of insurance. The result of this analysis is stated in Proposition 7:

Proposition 7. Consider a child who dislikes providing LTC to her parent and assume that $w<$ $(1-\gamma) \delta$ holds after a purchase of a marginal amount of insurance. Purchasing a positive amount of insurance is in the interest of the parent more often when insurance benefits are fixed than when insurance benefits are proportional.

Proposition 7 will be proved if we can show the following:

- if $\left.\frac{\partial E U^{p}}{\partial B}\right|_{B=0} \leq 0$, then $\left.\frac{\partial E U^{p}}{\partial \gamma}\right|_{\gamma=0}<0$ (i.e. if the parent does not find it beneficial to buy a positive amount of insurance when insurance benefits are fixed, he will definitely not find it beneficial when 
benefits are proportional)

and

- if $\left.\frac{\partial E U^{p}}{\partial \gamma}\right|_{\gamma=0} \geq 0$, then $\left.\frac{\partial E U^{p}}{\partial B}\right|_{B=0}>0$ (i.e. if the parent finds it beneficial to buy insurance when benefits are proportional, he will definitely find it beneficial when benefits are fixed)

but the reverse is not true.

This is indeed shown in the Appendix.

To summarize, we have seen that, in the case when the child dislikes providing care, insurance is more likely to be in the interest of the parent when benefits are fixed rather than proportional. To put it differently, the non-purchase of LTC insurance is more likely in the case of proportional benefits. The reason for this result is the fact that, as seen in the analysis of the child, the phenomenon of intra-family moral hazard is less pronounced with fixed insurance benefits. With fixed benefits, the purchase of insurance has less "severe" consequences to the parent in terms of the reduction in the child's caregiving, which makes it more likely that these negative consequences will be offset by the positive effects of insurance.

\section{Case 2: the child likes providing care $\left(\frac{\partial u^{c}}{\partial Q}>0\right)$}

To avoid corner solutions, the analysis in this case is focused on relatively high levels of the child's wage, i.e. $w>\delta$.

To compare the parent's decisions of whether to buy a positive amount of insurance or not, we can again look at $\left.\frac{\partial E U^{p}}{\partial \gamma}\right|_{\gamma=0}$ and $\left.\frac{\partial E U^{p}}{\partial B}\right|_{B=0}$. Their expressions in this case are the same as given by (31) and (32), but the signs of $\left.\frac{\partial Q}{\partial \gamma}\right|_{\gamma=0}$ and $\left.\frac{\partial Q}{\partial B}\right|_{B=0}$ are now different. As seen in the analysis of the child, in this case insurance with fixed benefits always induces the child to increase her caregiving (Proposition 4), which means that $\left.\frac{\partial Q}{\partial B}\right|_{B=0}$ is now always positive. Again focusing on the cases where the first term of the derivatives is positive, this implies that $\left.\frac{\partial E U^{p}}{\partial B}\right|_{B=0}$ is now always positive as well and so the parent will always decide to buy a positive amount of insurance when benefits are fixed.

On the other hand, from Proposition 4 we know that with proportional benefits the effect of insurance on $Q$ is ambiguous. This implies that the sign of $\left.\frac{\partial E U^{p}}{\partial \gamma}\right|_{\gamma=0}$ is not clear. If $\left.\frac{\partial Q}{\partial \gamma}\right|_{\gamma=0} \geq 0,\left.\frac{\partial E U^{p}}{\partial \gamma}\right|_{\gamma=0}$ is 
positive and thus the parent will always buy a positive amount of insurance with proportional benefits as well. However, if $\left.\frac{\partial Q}{\partial \gamma}\right|_{\gamma=0}<0$, it is possible that $\left.\frac{\partial E U^{p}}{\partial \gamma}\right|_{\gamma=0}$ will turn negative, which means that in the case of proportional benefits it might be optimal for the parent not to purchase insurance at all.

The above can be summarized as follows:

Proposition 8. Consider a child who likes providing LTC to her parent and has a wage $w>\delta$. Assume also that the parent would buy insurance in the absence of an impact on the child's caregiving. When insurance has an impact on the child's caregiving, purchasing a positive amount of insurance is always in the interest of the parent when insurance benefits are fixed. With proportional insurance benefits, purchasing a positive amount of insurance is not always in the interest of the parent.

Proposition 8 flows naturally from the analysis of the child. Since, when the child likes providing care, fixed benefits completely eliminate intra-family moral hazard, the intra-family moral hazard related motive for the non-purchase of insurance simply disappears. Even more than that can be said: since in this case insurance even induces the child to increase her caregiving, the impact on the child becomes an argument pushing for (and not against as before) the purchase of insurance. Thus, even in the case when the parent would not buy insurance in the absence of an effect on the child, he might decide to purchase insurance precisely because of the positive impact on the child's caregiving. It is true that the same situation is possible with proportional benefits as well; however, with proportional benefits the possibility of intra-family moral hazard still remains, in which case the parent might be discouraged from purchasing insurance. Therefore, as in the case when the child dislikes providing care, we can conclude here as well that insurance is in the interest of the parent more often when benefits are fixed than when they are proportional.

\section{Conclusion}

The paper has raised and explored formally the idea that, in the context of intra-family moral hazard suggested by Pauly (1990), fixed insurance benefits might have a somewhat different impact on the behavior of children and parents than benefits proportional to LTC expenditure do. In particular, the paper has studied and compared the effects that insurance with fixed and proportional benefits has on 
the caregiving choice of a child and examined how these different effects influence the insurance purchase decisions of a parent who values the care provided by his child.

The results of the paper formally confirm the intuitive hypothesis that fixed insurance benefits should help to limit intra-family moral hazard. Indeed, in the case of a child who dislikes providing care, the paper finds that even though the child's caregiving is decreasing in insurance coverage with both types of benefits, the same amount of LTC insurance purchased by the parent results in more care provided by the child when insurance benefits are fixed rather than proportional. Thus, with fixed benefits the child reduces her caregiving less drastically. The amount of the child's care provision is greater with fixed than with proportional benefits when she likes providing care as well. In that case, fixed benefits even eliminate intra-family moral hazard completely, whereas the effect of proportional benefits is ambiguous.

These differences between fixed and proportional benefits have direct consequences on the parent's insurance decisions. Indeed, even though generally the parent might find insurance undesirable with both types of benefits, it is shown that purchasing a positive amount of insurance is more likely to be in the parent's interest when benefits are fixed rather than proportional. In other words, the non-purchase of LTC insurance is more likely with proportional benefit schemes.

The findings of the paper can be of some use to public policy as well. Zweifel and Strüwe (1998) have raised concerns about the welfare effects that are/could be caused by a compulsory social LTC insurance. In particular, since (because of intra-family moral hazard) parents might in many cases find private LTC insurance undesirable, imposing a compulsory social insurance might go against the interests of many parents. The analysis in this paper has, however, shown that insurance can be made more acceptable to parents by using fixed benefit schemes. In addition to this, children are better-off with fixed benefits as well. Intra-family moral hazard thus pushes for favouring public schemes with fixed rather than proportional benefits.

Finally, it should be reminded that the analysis in this paper is focused on the context of intrafamily moral hazard and thus abstracts from other factors and situations that could potentially modify the conclusions about the desirability of fixed and proportional insurance benefits. For instance, while the model is limited to a single level of LTC needs, one could argue that the presence of uncertainty about these needs would enhance the desirability of proportional insurance benefits. In such a more 
comprehensive framework one could thus expect the parent to find himself facing a tradeoff between a better protection against the uncertainty offered by proportional insurance and a limitation of intrafamily moral hazard achieved with fixed benefits. On the other hand, it should be noted that fixed benefit schemes often allow the size of the benefits to depend on the degree of the insured's dependence, which (at least to some extent) improves their performance in terms of protection against uncertain needs and increases their chances to be overall preferred to proportional ones.

\section{References}

[1] Brown, Jeffrey R. and Amy Finkelstein. "Insuring Long-Term Care in the United States". Journal of Economic Perspectives, Vol. 25, No. 4 (2011), pp. 119-142.

[2] Colombo, Francesca, Ana Llena-Nozal, Jérôme Mercier and Frits Tjadens. "Help Wanted? Providing and Paying for Long-Term Care". OECD Health Policy Studies, OECD Publishing, 2011.

[3] Courbage, Christophe and Nolwenn Roudaut. "Empirical Evidence on Long-Term Care Insurance Purchase in France". The Geneva Papers on Risk and Insurance - Issues and Practice, Vol. 33 (2008), pp. $645-658$.

[4] Courbage, Christophe and Peter Zweifel. "Two-Sided Intergenerational Moral Hazard, Long-Term Care Insurance, and Nursing Home Use". Journal of Risk and Uncertainty, Vol. 43 (2011), pp. 65-80.

[5] Cremer, Helmuth, Pierre Pestieau and Grégory Ponthière. "The Economics of Long-Term Care: A Survey". Nordic Economic Policy Review, No. 2 (2012), pp. 107-148.

[6] Cutler, David. "Why Doesn't the Market Fully Insure Long-Term Care?". NBER Working Paper No. 4301 (1993).

[7] Duran, Romain and Lucie Taleyson. "Les Raisons du Succès de l'Assurance Dépendance en France". Risques - Les Cahiers de l'Assurance, Vol. 55 (2003), pp. 115-120. 
[8] European Commission. "The 2009 Ageing Report: Economic and Budgetary Projections for the EU27 Member States (2008-2060)". Joint Report prepared by the European Commission (DG ECFIN) and the Economic Policy Committee (AWG), 2009.

[9] Kemper, Peter and Christopher M. Murtaugh. "Lifetime Use of Nursing Home Care". The New England Journal of Medicine, Vol. 324, No. 9 (1991), pp. 595-600.

[10] Kessler, Denis. "The Long-Term Care Insurance Market". The Geneva Papers on Risk and Insurance - Issues and Practice, Vol. 33 (2008), pp. 33-40.

[11] Norton, Edward C. "Long-Term Care", in A.J. Culyer and J.P. Newhouse (Eds): Handbook of Health Economics, Vol. 1, Chapter 17 (2000), pp. 955-994.

[12] Pauly, Mark V. "The Rational Nonpurchase of Long-Term Care Insurance". Journal of Political Economy, Vol. 98, No. 1 (1990), pp. 153-168.

[13] Pauly, Mark V. "Almost Optimal Social Insurance for Long-Term Care", in R. Eisen and P. Sloan (Eds): Long-Term Care: Economic Issues and Policy Solutions, London, Kluwer, 1996.

[14] Pestieau, Pierre and Grégory Ponthière. "The Long-Term Care Insurance Puzzle", in J. Costa-Font and C. Courbage (Eds): Financing Long-Term Care in Europe: Institutions, Markets and Models, Palgrave Macmillan, 2011.

[15] Sloan, Frank A. and Edward C. Norton. "Adverse Selection, Bequests, Crowding Out, and Private Demand for Insurance: Evidence from the Long-Term Care Insurance Market". Journal of Risk and Uncertainty, Vol. 15 (1997), pp. 201-219.

[16] Taleyson, Lucie. "L’Assurance Dépendance Privée. Comparaisons Internationales". Newsletters Techniques SCOR, 9 (2003).

[17] Zweifel, Peter and Wolfram Strüwe. "Long-Term Care Insurance and Trust Saving in a TwoGeneration Model“, in R. Eisen and P. Sloan (Eds): Long-Term Care: Economic Issues and Policy Solutions, London, Kluwer, 1996. 
[18] Zweifel, Peter and Wolfram Strüwe. "Long-Term Care Insurance in a Two-Generation Model". The Journal of Risk and Insurance, Vol. 65, No. 1 (1998), pp. 13-32.

\section{Appendix: Proof of Proposition 7}

Let us start by proving the first statement: if $\left.\frac{\partial E U^{p}}{\partial B}\right|_{B=0} \leq 0$, then $\left.\frac{\partial E U^{p}}{\partial \gamma}\right|_{\gamma=0}<0$.

Assume that $\left.\frac{\partial E U^{p}}{\partial B}\right|_{B=0} \leq 0$, that is:

$$
\pi(1-\pi)\left(\left.\frac{\partial u^{p}}{\partial W_{p}^{D}}\right|_{B=0}-\left.\frac{\partial v^{p}}{\partial W_{p}^{I}}\right|_{B=0}\right) \leq-\left.\pi \frac{\partial Q}{\partial B}\right|_{B=0}\left(\left.\frac{\partial u^{p}}{\partial Q}\right|_{B=0}+\left.\delta \frac{\partial u^{p}}{\partial W_{p}^{D}}\right|_{B=0}\right)
$$

We need to show that $\left.\frac{\partial E U^{p}}{\partial \gamma}\right|_{\gamma=0}<0$, that is:

$$
\pi(1-\pi) \delta(\bar{L}-Q)\left(\left.\frac{\partial u^{p}}{\partial W_{p}^{D}}\right|_{\gamma=0}-\left.\frac{\partial v^{p}}{\partial W_{p}^{I}}\right|_{\gamma=0}\right)<-\left.\pi \frac{\partial Q}{\partial \gamma}\right|_{\gamma=0}\left(\left.\frac{\partial u^{p}}{\partial Q}\right|_{\gamma=0}+\left.\delta \frac{\partial u^{p}}{\partial W_{p}^{D}}\right|_{\gamma=0}\right)
$$

Note that $\left.\frac{\partial u^{p}}{\partial W_{p}^{D}}\right|_{B=0}=\left.\frac{\partial u^{p}}{\partial W_{p}^{D}}\right|_{\gamma=0}$ and $\left.\frac{\partial v^{p}}{\partial W_{p}^{I}}\right|_{B=0}=\left.\frac{\partial v^{p}}{\partial W_{p}^{I}}\right|_{\gamma=0}$.

If in inequality (34) we replace $\pi(1-\pi)\left(\left.\frac{\partial u^{p}}{\partial W_{p}^{D}}\right|_{\gamma=0}-\left.\frac{\partial v^{p}}{\partial W_{p}^{I}}\right|_{\gamma=0}\right)$ by $-\left.\pi \frac{\partial Q}{\partial B}\right|_{B=0}\left(\left.\frac{\partial u^{p}}{\partial Q}\right|_{B=0}+\left.\delta \frac{\partial u^{p}}{\partial W_{p}^{D}}\right|_{B=0}\right)$, we get:

$$
-\left.\delta(\bar{L}-Q) \pi \frac{\partial Q}{\partial B}\right|_{B=0}\left(\left.\frac{\partial u^{p}}{\partial Q}\right|_{B=0}+\left.\delta \frac{\partial u^{p}}{\partial W_{p}^{D}}\right|_{B=0}\right)<-\left.\pi \frac{\partial Q}{\partial \gamma}\right|_{\gamma=0}\left(\left.\frac{\partial u^{p}}{\partial Q}\right|_{\gamma=0}+\left.\delta \frac{\partial u^{p}}{\partial W_{p}^{D}}\right|_{\gamma=0}\right)
$$

If we show that (35) holds, then (34) holds as well (because of (33)).

Since $\left.\frac{\partial u^{p}}{\partial Q}\right|_{B=0}=\left.\frac{\partial u^{p}}{\partial Q}\right|_{\gamma=0}$ and $\left.\frac{\partial u^{p}}{\partial W_{p}^{D}}\right|_{B=0}=\left.\frac{\partial u^{p}}{\partial W_{p}^{D}}\right|_{\gamma=0}$, inequality (35) is equivalent to

$$
\begin{gathered}
\delta(\bar{L}-Q)\left(-\left.\frac{\partial Q}{\partial B}\right|_{B=0}\right)<\left(-\left.\frac{\partial Q}{\partial \gamma}\right|_{\gamma=0}\right) \\
\Longleftrightarrow \\
\delta(\bar{L}-Q)<\left.\frac{\partial Q}{\partial \gamma}\right|_{\gamma=0} /\left.\frac{\partial Q}{\partial B}\right|_{B=0}
\end{gathered}
$$

We get from (16) and (17):

$$
\left.\frac{\partial Q}{\partial \gamma}\right|_{\gamma=0}=-\left.\delta \frac{\partial \tilde{Q}}{\partial p_{p r}}\right|_{\gamma=0}+\left.\frac{\partial Q}{\partial I_{p r}}\right|_{\gamma=0} \delta(1-\pi)(\bar{L}-Q)
$$

and

$$
\left.\frac{\partial Q}{\partial B}\right|_{B=0}=\left.\frac{\partial Q}{\partial I_{f}}\right|_{B=0}(1-\pi)
$$


Using the fact that $\left.\frac{\partial Q}{\partial I_{p r}}\right|_{\gamma=0}=\left.\frac{\partial Q}{\partial I_{f}}\right|_{B=0}$, we have

$$
\left.\frac{\partial Q}{\partial \gamma}\right|_{\gamma=0} /\left.\frac{\partial Q}{\partial B}\right|_{B=0}=\underbrace{\left[\frac{-\left.\delta \frac{\partial \tilde{Q}}{\partial p_{p r}}\right|_{\gamma=0}}{\left.\frac{\partial Q}{\partial I_{f}}\right|_{B=0}(1-\pi)}\right]}_{(+)}+\delta(\bar{L}-Q)
$$

Thus, (37) clearly holds, which means that (35) and thus (34) holds.

Now let us prove the second statement: if $\left.\frac{\partial E U^{p}}{\partial \gamma}\right|_{\gamma=0} \geq 0$, then $\left.\frac{\partial E U^{p}}{\partial B}\right|_{B=0}>0$.

Assume that $\left.\frac{\partial E U^{p}}{\partial \gamma}\right|_{\gamma=0} \geq 0$, that is:

$$
\pi(1-\pi) \delta(\bar{L}-Q)\left(\left.\frac{\partial u^{p}}{\partial W_{p}^{D}}\right|_{\gamma=0}-\left.\frac{\partial v^{p}}{\partial W_{p}^{I}}\right|_{\gamma=0}\right) \geq-\left.\pi \frac{\partial Q}{\partial \gamma}\right|_{\gamma=0}\left(\left.\frac{\partial u^{p}}{\partial Q}\right|_{\gamma=0}+\left.\delta \frac{\partial u^{p}}{\partial W_{p}^{D}}\right|_{\gamma=0}\right)
$$

We need to show that $\left.\frac{\partial E U^{p}}{\partial B}\right|_{B=0}>0$, that is:

$$
\pi(1-\pi)\left(\left.\frac{\partial u^{p}}{\partial W_{p}^{D}}\right|_{B=0}-\left.\frac{\partial v^{p}}{\partial W_{p}^{I}}\right|_{B=0}\right)>-\left.\pi \frac{\partial Q}{\partial B}\right|_{B=0}\left(\left.\frac{\partial u^{p}}{\partial Q}\right|_{B=0}+\left.\delta \frac{\partial u^{p}}{\partial W_{p}^{D}}\right|_{B=0}\right)
$$

(41) is equivalent to

$$
\pi(1-\pi)\left(\left.\frac{\partial u^{p}}{\partial W_{p}^{D}}\right|_{\gamma=0}-\left.\frac{\partial v^{p}}{\partial W_{p}^{I}}\right|_{\gamma=0}\right) \geq-\left.\frac{\pi}{\delta(\bar{L}-Q)} \frac{\partial Q}{\partial \gamma}\right|_{\gamma=0}\left(\left.\frac{\partial u^{p}}{\partial Q}\right|_{\gamma=0}+\left.\delta \frac{\partial u^{p}}{\partial W_{p}^{D}}\right|_{\gamma=0}\right)
$$

If we show that

$$
-\left.\frac{\pi}{\delta(\bar{L}-Q)} \frac{\partial Q}{\partial \gamma}\right|_{\gamma=0}\left(\left.\frac{\partial u^{p}}{\partial Q}\right|_{\gamma=0}+\left.\delta \frac{\partial u^{p}}{\partial W_{p}^{D}}\right|_{\gamma=0}\right)>-\left.\pi \frac{\partial Q}{\partial B}\right|_{B=0}\left(\left.\frac{\partial u^{p}}{\partial Q}\right|_{B=0}+\left.\delta \frac{\partial u^{p}}{\partial W_{p}^{D}}\right|_{B=0}\right)
$$

then (42) holds.

(44) is equivalent to

$$
\begin{gathered}
-\left.\frac{1}{\delta(\bar{L}-Q)} \frac{\partial Q}{\partial \gamma}\right|_{\gamma=0}>-\left.\frac{\partial Q}{\partial B}\right|_{B=0} \\
\Longleftrightarrow \\
\left.\frac{\partial Q}{\partial \gamma}\right|_{\gamma=0} /\left.\frac{\partial Q}{\partial B}\right|_{B=0}>\delta(\bar{L}-Q)
\end{gathered}
$$

which we have shown to be true above.

It can be easily seen that the reverse of the two claims is not true. 